\title{
Molecular Survey of Mycoplasma haemofelis and 'Candidatus Mycoplasma Haemominutum' Infection in Cats in Yamaguchi and Surrounding Areas
}

\author{
Hisashi INOKUMA ${ }^{1)}$, Shoko TAROURA ${ }^{1)}$, Masaru OKUDA ${ }^{1)}$, Masaharu HISASUE ${ }^{2)}$, Kazuhito ITAMOTO ${ }^{1)}$, \\ Satoshi UNE ${ }^{1)}$, Munekazu NAKAICHI ${ }^{1)}$ and Yasuho TAURA ${ }^{1)}$ \\ ${ }^{1)}$ Faculty of Agriculture, Yamaguchi University, Yamaguchi, Yamaguchi 753-8515 and ${ }^{2)}$ School of Veterinary Medicine, Azabu \\ University, Sagamihara, Kanagawa 229-8501, Japan
}

(Received 5 February 2003/Accepted 26 March 2004)

ABSTRACT. A molecular survey of hemoplasma (Mycoplasma haemofelis and 'Candidatus Mycoplasma haemominutum') in Yamaguchi Prefecture and surrounding areas was performed by using molecular methods. PCR-RFLP with HindIII revealed that 2 cats were infected with $M$. haemofelis, and 16 with 'C. Mycoplasma haemominutum' among 102 randomly selected cats. Partial 16S rRNA gene sequences of $M$. haemofelis and 'C. Mycoplasma haemominutum' determined in this study showed percent similarities of 98.3-99.8\% and 96.4$100 \%$, respectively, with those from other countries. Hemoplasma infections were more frequently detected in free-roaming cats than inside cats. Also, the status of FeLV infection was another significant risk factor for hemoplasma infection.

KEY wORDS: 'Candidatus Mycoplasma haemominutum', feline, Mycoplasma haemofelis.

J. Vet. Med. Sci. 66(8): 1017-1020, 2004

The feline hemoplasmas, Mycoplasma haemofelis and 'Candidatus Mycoplasma haemominutum', were previously ascribed to Haemobartonella felis strains Ohio and California, respectively [13, 14], which cause hemolytic anemia, thrombocytopenia, fever and jaundice [3, 10]. Hemoplasma infection is usually diagnosed through the detection of the parasite on the surface of erythrocytes in blood smears stained with Giemsa and viewed under a microscope [1]. However, it is often difficult to confirm the infection, as the organisms resemble Howell-Jolly bodies or background debris [2]. It is even more difficult to distinguish $M$. haemofelis and ' $C$. Mycoplasma haemominutum' morphologically. A number of molecular techniques, including polymerase chain reaction (PCR) and restriction fragment length polymorphism (RFLP) analysis, have recently been developed for feline hemoplasmas infection, and widely used for both diagnosis and epidemiological studies in the U.K. [16, 17], Spain [5], U.S.A. [7, 12], Israel [9], and Australia [4]. However, limited numbers of studies on feline hemoplasma infection in Japan have been published. Thus, the aim of the present study was to analyze the infection of hemoplasma in cats in Yamaguchi and surrounding area by using molecular methods.

A total of 102 feline patients were randomly selected from cats that presented at the Veterinary Teaching Hospital, Yamaguchi University, from March 2002 to March 2003. Twelve of 102 cats were presented for physical examination or vaccination, and did not show any clinical problems. Thus these cats were categorized as a healthy group. The rest of the 90 cats showed one or more diseases. Blood treated with the anticoagulant EDTA was collected for PCR to detect hemoplasma. Clinical symptoms were recorded at the time of blood collection. Infection of feline leukemia virus (FeLV) and feline immunodeficiency virus (FIV) was occasionally checked using a SNAP FeLV/FIV
Combo kit (IDEXX, U.S.A.). The significance of individual factors (age, sex, roaming, inside/outside, health condition, FIV and FeLV infection) as determinants for hemoplasma infection was investigated by maximum likelihood estimates of the chi square test derived from contingency table analysis. The critical probability was set at $\mathrm{p}=0.05$.

Total DNA was extracted from the packed red blood cell fraction of each feline sample by a method described previously [11]. For a screening purpose, PCR amplification was performed in a $25-\mu l$ reaction mixture containing $5 \mu l$ of each DNA template with the primer set of $H$. felis $\mathrm{F}$ and $H$. felis $\mathrm{R}$ described by Jensen et al. [12], which can amplify both $M$. haemofelis and ' $C$. Mycoplasma haemominutum'. These primers produce a 170-bp fragment in M. haemofelis and a 193-bp fragment in ' $C$. Mycoplasma haemominutum'. Positive DNA controls for $M$. haemofelis and ' $C$. Mycoplasma haemominutum' were kindly supplied by Dr. S. Tasker (University of Bristol, UK). To confirm the nature of the amplification products of both $M$. haemofelis and ' $C$. Mycoplasma haemominutum,' the PCR products were digested with HindIII (New England Biolabs, U.S.A.) according to the recommendations of the manufacturer. The PCR products and digestion products were separated by using $3 \%$ agarose gel electrophoresis. Partial 1 sequence of $16 \mathrm{~S}$ rRNA gene, including the divergent region near the 5'end, were then determined for the screening-positive samples. A primer set, fD1 [19] and Hf513R (5'-ACG CCC AAT AAA TCC GAA TAA-3'), were used for the amplification, and nucleotide sequences of the PCR products were determined and analyzed by the method previously reported [11]. The GenBank accession numbers of 16S rRNA gene sequences used to analyze the percent identities and to construct a phylogenetic tree were as follows: M. haemofelis strains Australia 1: AY150976, Australia 2: AY150977, 
UK5: AY150984, UK6: AY150985, France: AY150972, Illinois U.S.A.: U95297, North Carolina U.S.A.: AY069948, Oklahoma U.S.A.: AF178677, Ohio-Florida U.S.A.: U88563, South Africa: AF548631, 'Candidatus Mycoplasma haemominutum' strains UK 1: AY150980, UK 2: AY 150981, Israel: AY 150974, South Africa: AY150979, Birmingham U.S.A.: AF271154, California U.S.A.: U88564, Mycoplasma haemocanis strain Illinois: AF197337, Mycoplasma haemomuris: U82963, Mycoplasma suis: U88565, Mycoplasma ovis: AF338268 and Mycoplasma pneumoiae: M29061. The nucleotide sequences of the $16 \mathrm{~S}$ rRNA gene of $M$. haemofelis detected from cats in this study have been deposited in the GenBank database under the following accession numbers as follows: Yamaguchi 4806; AY529629 and Yamaguchi 6287; AY529632. The accession numbers of the partial sequences of the 16S rRNA gene of ' $C$. Mycoplasma haemominutum' determined in this study were as follows: Yamaguchi 6167; AY529628, Yamaguchi 5091; AY529630, Yamaguchi 6424; AY529633, Yamaguchi 6532; AY529635, Yamaguchi 6571; AY529637, Yamaguchi 6584; AY529638, Yamaguchi 6683; AY 529679, Yamaguchi 6763; AY529640, Hiroshima 6439; AY529634, Fukuoka 5884; AY529631 and Fukuoka 6566; AY529636.

Hemoplasma was detected in $18(17.7 \%)$ out of 102 randomly selected cats by PCR screening. The healthy population $(n=12)$ failed to amplify the target gene, while the positive ratio in currently ill cats was $20.0 \%$ (18 among 90 cats). Subsequent RFLP with HindIII yielded fragments of
76 and $117 \mathrm{bp}$ for ' $C$. Mycoplasma haemominutum,' and no cut in M. haemofelis (Fig. 1). Digestion of the PCR products with HindIII revealed that 2 out of 18 positive cats were infected with $M$. haemofelis, and the remaining 16 with ' $C$. Mycoplasma haemominutum'. One (Yamaguchi 6287) cat infected with $M$. haemofelis, showed typical clinical symptoms of feline hemobartonellosis, including hemolytic anemia, anorexia, jaundice and haematuria. But others did not show any signs of hemobartonellosis. Watanabe et al. reported that among 18 cats infected with hemoplasma in Japan, 12 cats $(67 \%)$ were infected with M. haemofelis, 4 (22\%) with ' $C$. Mycoplasma haemominutum', and 2 with both pathogens [18]. They used cats that showed clinical symptoms of hemoplasma infection. This may have caused the difference of the positive ratio in the two studies. As the pathogenesis of $M$. haemofelis is generally more severe than that of ' $C$. Mycoplasma haemominutum' $[6,20]$, the percentage of cats positive for $M$. haemofelis would be higher in symptomatic cats than in healthy or non-symptomatic cats.

Partial 16S rRNA gene sequences, approximately $500 \mathrm{bp}$ in length were successfully determined for 13 of 18 hemoplasma-positive cats. The sequences of 2 isolates of $M$. haemofelis obtained from cats in Yamaguchi Prefecture were $100 \%$ identical with each other and showed percent identities of 98.3 to $99.8 \%$ with those of isolates from U.S.A., Europe, Australia, Israel and South Africa. The phylogenetic analysis showed that the 2 sequences of $M$. haemofelis determined in this study belong to the same

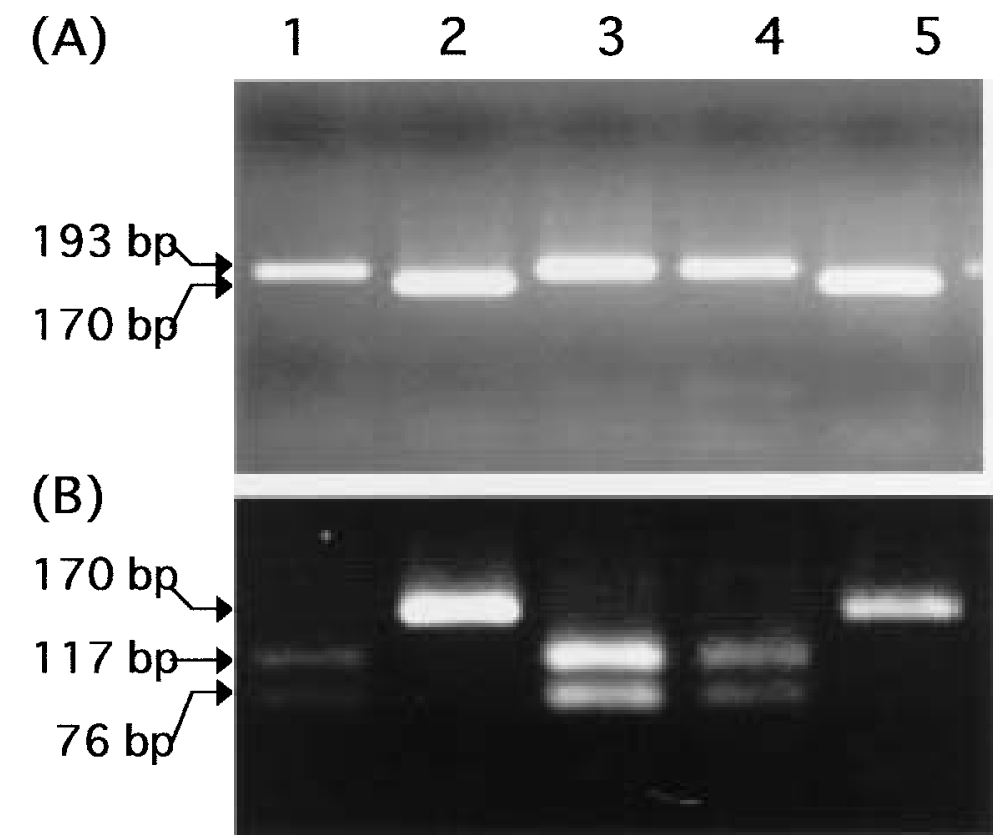

Fig. 1. Results of PCR and RFLP from 5 hemoplasma-positive cats. (A) Screening by PCR produced a 193-bp fragment for ' $C$. Mycoplasma haemominutum.' (Lanes 1, 3 and 4) and a 170-bp fragment for M. haemofelis (Lanes 2 and 5). (B) Subsequent RFLP analysis with HindIII yielded fragments of 76 and 117 bp for ' $C$. Mycoplasma haemominutum,' (Lanes 1, 3 and 4) and no cut in M. haemofelis (Lanes 2 and 5). 


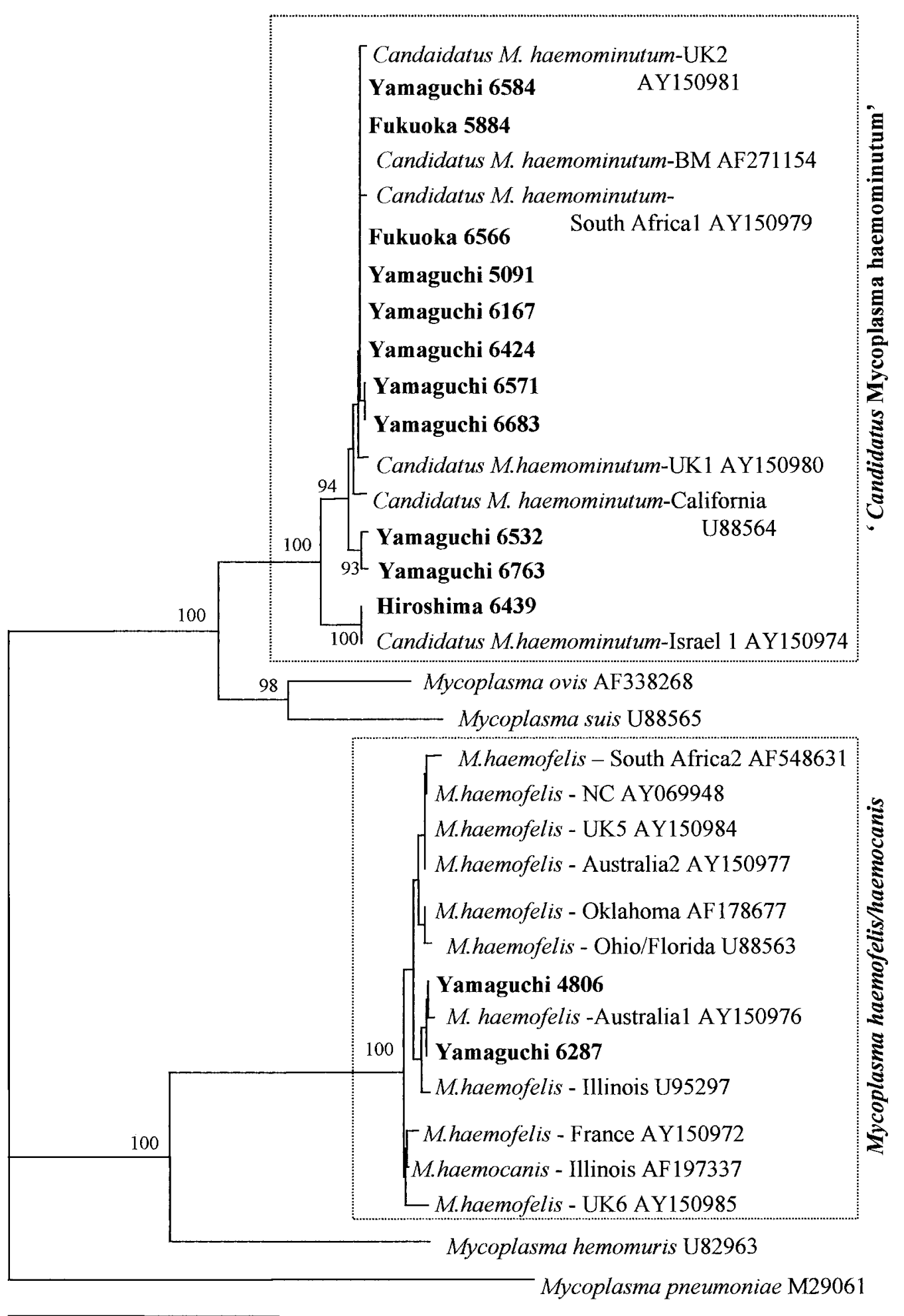

Fig. 2. Phylogenetic relationship of various hemoplasmas based on partial sequences of the 16S rRNA gene. The neighborjoining method was used to construct the phylogenetic tree with the Clustal W program. The scale bar represents $10 \%$ divergence. The numbers at nodes are the proportions of 100 bootstrap resamplings that support the topology shown. 
Table 1. Risk factor analysis for hemoplasma infection in cats

\begin{tabular}{lcccc}
\hline \multicolumn{1}{c}{ Factors } & $\begin{array}{c}\text { Numbers } \\
\text { of cats }\end{array}$ & \multicolumn{3}{c}{ Numbers of cats infected } \\
& & & & \\
\hline Roaming & 36 & 0 & 14 & 35 \\
In & 52 & 2 & 1 & 36 \\
Out & 14 & 0 & & 13 \\
$\quad$ Unknown & & & \\
$p$ value (chi square) & 0.0263 & & 2 & \\
& & & 8 & 1 \\
FeLV infection & & 1 & 6 & 29 \\
Positive & 4 & 1 & & \\
Negative & 63 & 0 & &
\end{tabular}

clade as M. haemofelis strains of other countries (Fig. 2). In contrast, 11 sequences of ' $C$. Mycoplasma haemominutum' determined in this study showed more variable percent identities: 96.4 to $100 \%$ with those of isolates from U.S.A., European countries, Israel and South Africa; however, they all belong to the same clade of ' $C$. Mycoplasma haemominutum' (Fig. 2).

Hemoplasma infections were more frequently detected in free-roaming cats than inside cats (Table $1, \mathrm{P}=0.0263$ ). This was also found in a previous study on risk factors for hemoplasma infection [8]. Transmission of feline hemoplasma by blood-sucking arthropods is considered by many to be the primary mode of transmission in cats [4], although such transmission has not been established experimentally except in the case of transmission of M. haemocanis by Rhipicephalus sanguineus [15]. It is also thought that transmission occur by cat-bite wounds [3]. The higher positive rate in free-roaming cats may reflect the higher possibility of exposure to blood-sucking arthropods or cat-bites. In addition, the status of FeLV infection was another significant risk factor for hemoplasma infection (Table $1, \mathrm{p}=0.0023$ ). There were no other significant differences in the frequency of hemoplasma infection according to age, sex, overall health condition, or FIV infection (data not shown). Although the reason why FeLV infection is related to hemoplasma infection in cats has not been determined yet, similar findings have been reported by others [7-9]. More recent reports have shown that both FeLV and FIV infections dramatically increase the possibility of hemoplasma infection [7, 9]. As only a small numbers of cats were used for the analysis in the present study, greater numbers of cats should be examined to clarify the relationship between virus infection and hemoplasma infection in cats in Japan.

ACKNOWLEDGMENTS. The authors would like to acknowledge the technical expertise of the DNA Core facility of the Center for Gene Research, Yamaguchi University, supported by a grant-in-aid from the Ministry of Education, Science, Sports and Culture of Japan. This work was supported in part by Merial Japan Ltd. and a Grant-in-Aid for Scientific Research from the Japan Society for the Promotion of Science (Grant No. 14360190).

\section{REFERENCES}

1. Bobade, P. A., Nash, A.S. and Rogerson, P. 1988. Vet. Rec. 122: $32-36$.

2. Bobade, P. A. and Nash, A.S. 1987. Vet. Parasitol. 26: 169172.

3. Breitschwerdt, E. B. 2000. Textbook of Veterinary Internal Medicine. 5th ed., W. B. Saunders, Philadelphia.

4. Clark, P., Foster, S. F. and Spencer, P. B. 2002. Aust. Vet. J. 80: 703-704.

5. Criado-Fornelio, A., Martinez-Marcos, A., Buling-Sarana, A. and Barba-Carretero, J. C. 2003. Vet. Microbiol. 93: 307-317.

6. Foley, J. E., Harrus, S., Poland, A., Chomel, B. and Pedersen, N. C. 1998. Am. J. Vet. Res. 59: 1581-1588.

7. George, J. W., Rideout, B. A., Griffey, S. M. and Pedersen, N. C. 2002. Am. J. Vet. Res. 63: 1172-1178.

8. Grindem, C. B., Corbett, W. T. and Tomkins, M. T. 1990. J. Am. Vet. Med. Assoc. 196: 96-99.

9. Harrus, S., Klement, E., Aroch, I., Stein, T., Bark, H., Lavy, E., mazaki-Tovi, M. and Baneth, G. 2002. Vet. Rec. 151: 82-85.

10. Harvey, J. W. and Gaskin, J. M. 1977. J. Am. Anim. Hosp. Assoc. 13: 28-38.

11. Inokuma, H.,Fujii, K., Okuda, M., Onishi, T., Beaufils, J-P., Raoult, D. and Brouqui, P. 2002. Clin. Diagn. Lab. Immunol. 9: 1132-1136.

12. Jensen, W. A., Lappin, M. R., Kamkar, S. and Reagen, W. J. 2001. Am. J. Vet. Res. 62: 604-608.

13. Messick, J. B., Walker, P. G., Raphael, W., Bernt, L. and Shi, X. 2002. Int. J. Syst. Evol. Microbiol. 52: 693-698.

14. Neimark, H., Johansson, K. E., Rikihisa, Y. and Tully, J. G. 2001. Int. J. Syst. Evol. Microbiol. 51: 891-899.

15. Seneviratna, P, Weerasinghe, and Ariyadasa, S. 1973. Res. Vet. Sci. 14: 112-114.

16. Tasker, S., Binns, S. H., Day, M. J., Gruffydd-Jones, T. J., Harbour, D. A., Helps, C. R., Jensen, W. A., Olver, C. S. and Lappin, M. R. 2003. Vet. Rec. 152: 193-198.

17. Tasker, S. Helps, C. R., Belford, C. J., Birtles, R, J., Day, M. J., Sparkes, A. H., Gruffydd-Jones, T. J. and Harbour, D. A. 2001. Vet. Microbiol. 81: 73-78.

18. Watanabe, M., Hisasue, M., Hashizaki, K., Furuichi, M., Ogata, M., Hisamatsu, S., Ogi, E., Hasegawa, M., Tsuchiya, R. and Yamada, T. 2003. J. Vet. Med. Sci. 65: 1111-1114.

19. Weisburg, W. G., Barns, S. M., Pelletier, D. A. and Lane, D. L., 1991. J. Bacteriol. 173: 697-703.

20. Westfall, D. S., Jensen, W. A., Reagen, W. J., Radecki, S. V. and Lappin, M.R. 2001. Am. J. Vet. Res. 62: 687-691. 